\title{
On the Field Emulation Techniques in Over-the-Air Testing: Experimental Throughput Comparison
}

\author{
Md. Suzan Miah, Dymtro Anin, Afroza Khatun, Katsuyuki Haneda, Member, IEEE, Lassi Hentila, \\ and Erkki T. Salonen, Member, IEEE
}

\begin{abstract}
In this letter, we compare two field emulation techniques, namely plane-wave synthesis (PWS) and prefading signal synthesis (PFS) for multiple-input-multiple-output over-theair (MIMO OTA) testing of mobile terminals in an anechoic chamber equipped with multiple probes. The comparison is performed in terms of measured throughput. The measurements were performed under urban microcellular scenario of the spatial channel model extended and at Long Term Evolution lower band 20. A practical mobile terminal is used as a test device. The throughput of four different browsing positions of the mobile terminal utilizing both emulation techniques has been measured. Results shows that at a fixed median throughput, the differences of the downlink channel power between the PFS and PWS techniques are small and are in the range of $0.2-1.0 \mathrm{~dB}$ for different browsing positions of the mobile terminal. The PWS and PFS techniques are equally capable of emulating the target field inside the test zone and are suitable for the MIMO OTA antenna testing based on anechoic chambers equipped with multiple probes.
\end{abstract}

Index Terms-Multiple-input-multiple-output (MIMO), MIMO over-the-air (OTA) testing, plane-wave synthesis (PWS), prefaded synthesis, radio channel, throughput.

\section{INTRODUCTION}

$\mathbf{M}$ ULTIPLE-INPUT-MULTIPLE-OUTPUT (MIMO) technique is an attractive technology to improve the performance of the wireless communication system. The performance, i.e., throughput and outage, of MIMO links depends not only on the antenna performance, but also on the propagation environment. Hence, testing of mobile terminal devices needs to consider both the multiple terminal antennas as well as the realistic propagation environment. Over-the-air (OTA) antenna

This work was supported in part by the Framework of Research Project MIMOTA-2 through the Finnish Funding Agency for Technology and Innovation (Tekes), in part by Microsoft, in part by Anite Oy (now Keysight), and in part by ETS-Lindgren. (Corresponding author: Md. Suzan Miah.)

M. Suzan Miah and K. Haneda are with the Department of Electronics and Nanoengineering, School of Electrical Engineering, Aalto University, 00076 Aalto, Finland (e-mail: md.miah@aalto.fi; katsuyuki.haneda@aalto.fi).

A. Khatun was with the Department of Electronics and Nanoengineering, School of Electrical Engineering, Aalto University, 00076, Aalto, Finland (e-mail: afroza.khatun2005@gmail.com).

D. Anin and E. T. Salonen are with the Center of Wireless Communications, University of Oulu, Oulu 90014, Finland (e-mail: dmytro.anin@oulu.fi; erkki.salonen@ee.oulu.fi).

L. Hentila is with the Keysight Technologies, Oulu 90590, Finland (e-mail: lassi.hentila@keysight.com). test methods have been widely used for multiantenna-equipped mobile devices.

Standardization works for the OTA test method to measure the MIMO performance have been intensively coordinated by the Cellular Telecommunications Industry Association (CTIA) and 3rd Generation Partnership Project (3GPP), where several different methods have been proposed [1]. One mandatory requirement of any test method is to emulate a realistic electromagnetic environment where mobile terminals operate in reality. Geometry-based channel models such as the spatial channel model (SCM) and SCM extended (SCME) provide base lines of the electromagnetic environment in the MIMO performance testing.

One of the proposed MIMO OTA antenna test methods in the standardization is the use of an anechoic chamber equipped with multiple probe antennas that emulate the intended electromagnetic environment inside a confined area of the chamber, which is called a test zone. We place a mobile terminal under test in the center of the test zone during the test. To emulate desired spatial and temporal characteristics of the intended electromagnetic environment defined by the channel model, two techniques have been reported in the literature. One technique is the prefaded signal synthesis (PFS) [2]-[4], which has been implemented in commercial tools. In [2], the feasibility of the PFS technique is experimentally validated in terms of power delay profile (PDP), power Doppler spectrum (PDS), and spatial correlation function (SCF) of the fields in the test zone. In [3], the PFS technique is verified through measurements of the PDS and SCF of the test zone field. The other potential technique is called a plane-wave synthesis (PWS) [5]-[10]. In [5], the PWS technique is validated experimentally through the accuracy of the synthesized test zone field in power, while the phase accuracy is discussed in [7]. In [3] and [10], PWS technique is verified by comparing the simulated and measured phase of the synthesized fields and the throughput, respectively.

Both methods are known to be equally capable for the MIMO OTA tests, though the different field emulation methods are used. However, there is no sufficient experimental evidence that the two methods performs equally fine, other than the work [9]; the work compares the two methods only on computer simulations of PDS, SCF, and temporal correlation function. As such, the simulation works in [9] give the initial idea about the justification of the two methods, but still lack the measurement verifications. Therefore, we in this letter provide verification of this compatibility based on measurements of the throughput. 


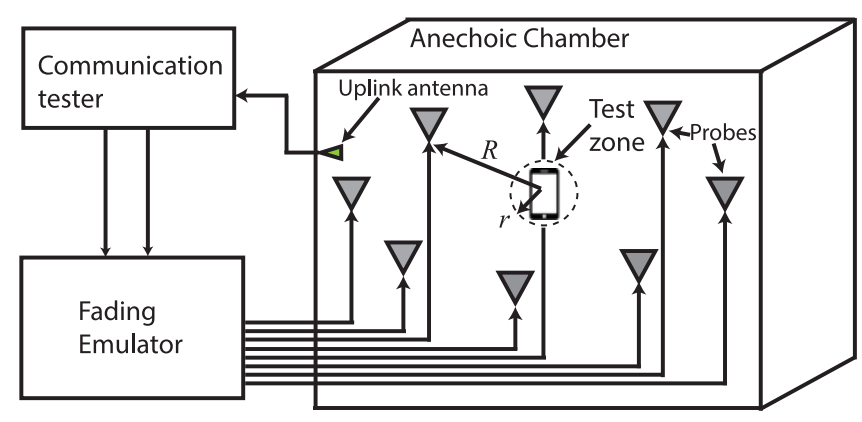

Fig. 1. Two-dimensional OTA antenna test ring with eight dual-polarized probes surrounding the antenna test zone with radius $r$. The probes are arranged on a circle with radius $R>r$.

Among possible figure-of-merit (FoM), throughput is the most important one in the MIMO OTA test of a multiantenna terminal as the end results of radio link performance [1], [11]. We, in particular, report the throughput against downlink (DL) power at a multiantenna mobile terminal under the identical setup of a multiprobe OTA chamber. The mobile terminal operates at the Long Term Evolution (LTE) downlink frequency of $806 \mathrm{MHz}$. The urban microcellular (UMi) scenario of the SCME [12] is considered as a target multipath channel. The comparison shows, for a fixed median throughput, the corresponding DL power of the fields generated by the PFS and PWS techniques are different by $1.0 \mathrm{~dB}$ at most for various browsing positions of the mobile terminal.

The rest of this letter is organized as follows. Section II describes the configuration of the anechoic-chamber-based multiprobe MIMO OTA antenna test Section III reports a measurement setup, procedure, and results, and Section IV concludes this letter.

\section{MIMO OTA ANTENNA Testing IN A CHAMBER}

\section{A. Probe Setup}

An illustration of the probe antennas arranged on a 2-D space in an anechoic chamber is shown in Fig. 1. The main components of the test system are a communication tester, a fading emulator, and a number of probe antennas. In Fig. 1, eight equally spaced dual-polarized antennas are distributed in the azimuth plane. The communication tester creates the DL transmit signals, which are fed to a multichannel fading emulator. The fading emulator generates the multipath environment. The output ports of the fading emulator are connected to the dualpolarized probe antennas. The radio frequency signals emitted from the probes to the air are controlled by the fading emulator to produce the intended electromagnetic environment inside the test zone. For the present 2-D test setup, a mobile terminal is at the center of the circular test zone with radius of $r$ and sends the data acknowledgement signals to an uplink (UL) communication antenna inside the chamber. The UL communication antenna is connected to the communication tester via a coaxial cable. It is required that the intended electromagnetic field should be accurately emulated inside the test zone, so that the multiantenna mobile terminal would experience the same propagation channels as it would be in the operation environment.

\section{B. Field Emulation Techniques}

1) PWS Technique: The PWS technique creates the intended electromagnetic field inside the test zone by exciting the multiple probes with proper complex weights, such that the total field from the probes resembles the intended field. The field is generated by superimposing multiple plane waves with specific angles of arrival (AoAs), Doppler shift, and magnitudes. The PWS technique reported in [5], [6], and [10] is based on the spherical wave theory [13], stating that any plane wave field in a test zone can be expressed by a finite sum of orthonormal wave modes. This spherical wave theory provides theoretical justification of the required number of probes as a result of its wellknown cutoff properties of the orthonormal modes. The PWS technique supports line-of-sight (LOS) paths between OTA antennas and controlled plolarization of paths. A drawback of the PWS technique is the requirement of phase calibration.

2) PFS Technique: The PFS technique generates the intended field in the test zone by a superposition of clusters, i.e., groups of multipath components [3], [4], [9]. The clusters are defined by their nominal AoAs, angular spreads of arrival, Doppler spectrum, and cross-polarization ratios. The PFS technique emulates the intended field by weighting the independent fading signals transmitted from the multiple probes. The signal weighting is made so that the power angular spectrum of the emulated field resembles that of the intended field. This leads to the accurate emulation of the spatial correlation characteristics inside the test zone [3], [9]. The PFS is recommended for standard models with wide clusters and Rayleigh fading or Ricean fading with single LOS component. The main benefit of the PFS compared to the PWS is that; phase calibration is not required.

\section{MEASUREMENTS}

\section{A. Setup and Procedure}

A measurement campaign was carried out in a MIMO OTA test setup at Anite Telecoms Oy, Finland (currently Keysight Technologies). It measures the throughput of a mobile terminal utilizing both the PWS and PFS techniques. The measurement system consists of an Anritsu MT 8820C communication tester, a Propsim F32 channel emulator, eight Vivaldi dual-polarized probe antennas, and a Nokia Lumia 635 mobile terminal at the center of the test zone as a test device.

Mobile terminal was placed on a turnable table, which allows us to align their orientations in the azimuth plane. The measurements were performed at $806 \mathrm{MHz}$, which is the LTE lower band 20. The modulation and coding scheme (MCS) 20 with 64 QAM DL modulation was considered in the measurements. The maximum DL theoretical throughput is $35.424 \mathrm{Mb} / \mathrm{s}$. Details of the measurement setup and parameters can be found in [11]. Fig. 2 shows a part of the measurement setup inside the anechoic chamber. Eight uniformly spaced probes on the azimuth ring out of three rings on the elevation domain, as shown in Fig. 2, were connected to the fading emulator, while the rest of 


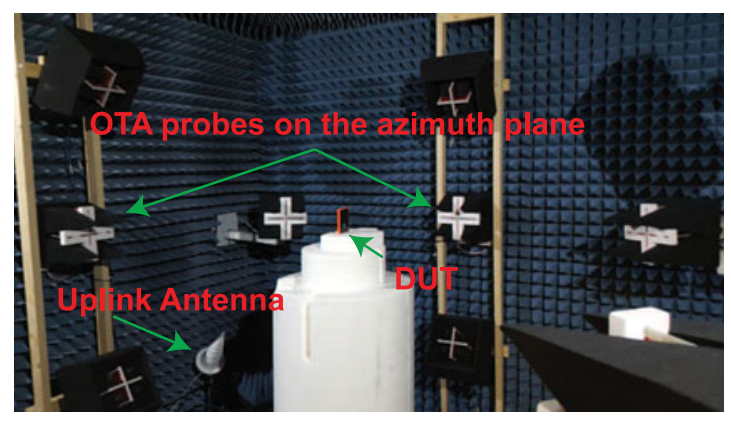

Fig. 2. Part of the probe setup in the anechoic chamber.

the probes on the other rings not in use were terminated during the measurement. The probe antennas were placed on the probe ring with the radius, $R$ in Fig. 1, of $1.5 \mathrm{~m}$ to ensure the far-field condition according to the study in [14]. Mobile terminal has two LTE-band-20 antennas, which are located at the lower back left and right side of the terminal, respectively, and have almost omnidirectional radiation patterns. Details of the antenna patterns can be found in [14]. The base-station antenna array considered in the channel emulator consists of colocated $\pm 45^{\circ}$ slanted dual-polarized dipoles fed with equal power [1].

Before performing the throughput measurements, the test equipment chain in the MIMO OTA antenna test setup was calibrated to ensure equal responses from each probe, in both magnitude and phase, by compensating for errors caused by setup such as probe misplacements and cable length variations. Reference electric and magnetic dipoles were used for the calibration of the vertical and horizontal components of the transmitted signal, respectively.

The PWS and PFS measurements were similar in terms of hardware requirements. As Propsim F32 channel emulator utilizes the PFS technique, the PFS measurements were performed without any change in the fading emulator. The power weights of the probe antennas were generated in the fading emulator. However, for the PWS measurements, the calculated complex probe weights based on spherical wave theory were imported to the fading emulator.

In the throughput measurement, the mobile terminal was placed at the center of the test zone, as shown in Fig. 2. The mobile terminal sends data acknowledgment signals back to the communication tester. The throughput was finally evaluated for both techniques at the communication tester as

$$
\mathrm{TP}=(1-\mathrm{BLER}) R_{\mathrm{b}} .
$$

where TP is the throughtput, BLER is the block error rate, and $R_{\mathrm{b}}$ is the transmit bit rate, which was $35.424 \mathrm{Mb} / \mathrm{s}$ defined by MCS 20. The DL signal-to-noise ratio at the mobile was controlled by varying the magnitude of the probe weights in fading emulator so that the total power per $15 \mathrm{kHz}$ subcarrier received by mobile terminal at the center of the chamber is between -95 and $-108 \mathrm{dBm}$. All measurements were performed under SCME UMi channel conditions.

In practical use of mobile terminals, they could operate in random orientations. The measurement was conducted in different

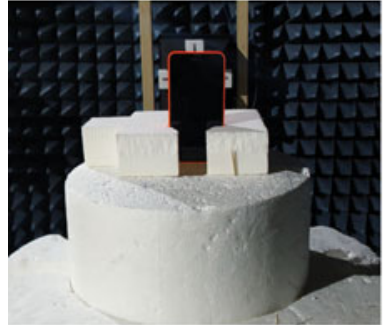

(a)

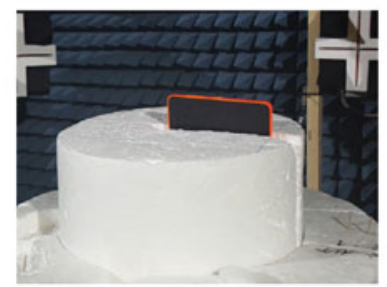

(c)

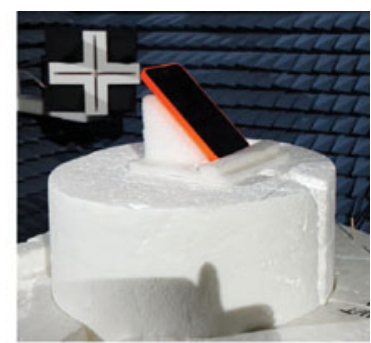

(b)

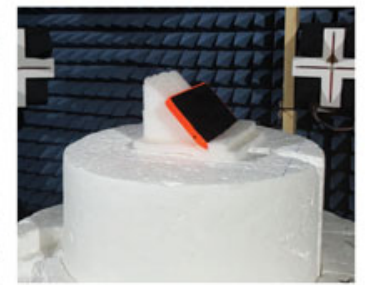

(d)
Fig. 3. Mobile terminal browsing positions: (a) Portrait $0^{\circ}$ tilted: P0, (b) Portrait $45^{\circ}$ tilted: P45, (c) Landscape $0^{\circ}$ : L0, and (d) Landscape $45^{\circ}$ : L45.

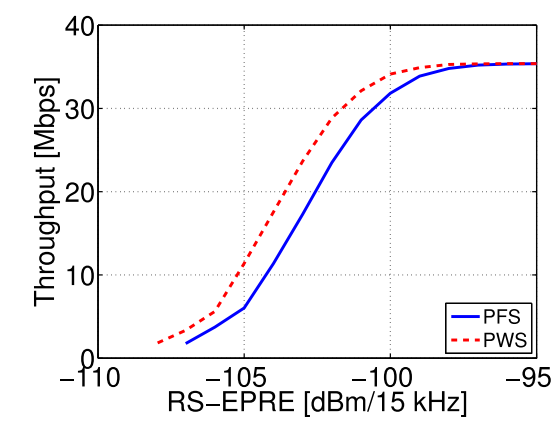

Fig. 4. Throughput comparison of the PWS and PFS techniques for the browsing position $\mathrm{P} 0$.

orientations corresponding to different browsing positions: portrait tilted by $0^{\circ}(\mathrm{P} 0)$, portrait tilted by $45^{\circ}(\mathrm{P} 45)$, landscape tilted by $0^{\circ}$ (L0), and landscape tilted by $45^{\circ}$ (L45), as defined in Fig. 3. For each browsing position, the mobile terminal was rotated on the azimuth plane with a step size of $\Delta \phi=30^{\circ}$, leading to 12 azimuthal steps in total. The throughput for each browsing position was obtained by averaging throughput over all the azimuthal rotation steps.

\section{B. Results}

Fig. 4 presents the measured throughput against DL power at the mobile, as expressed in the reference signal energy per resource element (RS-EPRE ${ }^{1}$ ). Results from both emulation techniques with the browsing positions $\mathrm{P} 0$, as shown in Fig. 3(a), are overlaid. The results show that the DL power is -102.9 and $-103.9 \mathrm{dBm}$ for the PFS and PWS techniques, for the median throughput, showing the difference of $1.0 \mathrm{~dB}$. Fig. 5

\footnotetext{
bit.

${ }^{1}$ The term RS-EPRE is specific in [1] and can be understood as the energy-per
} 


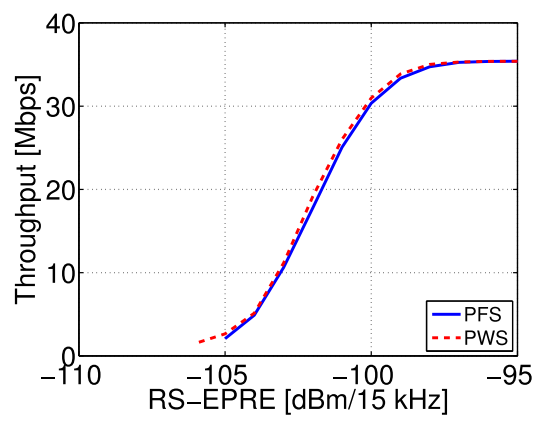

Fig. 5. Throughput comparison of the PWS and PFS techniques for the browsing position L45.

TABLE I

SuMMARY OF THE DL POWERS IN THE MIMO OTA TESTING OF A MoBILE PHONE TERMINAL

\begin{tabular}{lcccc}
\hline \hline Browsing positions & P0 & P45 & L0 & L45 \\
\hline DL power using PWS [dBm] & -103.9 & -101.4 & -103.8 & -102.3 \\
DL power using PFS [dBm] & -102.9 & -100.9 & -103.2 & -102.1 \\
Difference, $\Delta$ [dB] & 1.0 & 0.5 & 0.6 & 0.2 \\
\hline \hline
\end{tabular}

The powers with the PFS and PWS techniques as well as their differences are shown.

illustrates the throughput for the browsing position L45, as defined in Fig. 3(d). The difference of the DL power between these two techniques is $0.2 \mathrm{~dB}$ for the median throughput. Similar measurements were performed for other browsing positions in Fig. 3, showing that the differences of the DL power between the two techniques are at most $0.6 \mathrm{~dB}$ for the median throughput. A summary of DL power difference between the PFS and PWS techniques at the median throughput for different browsing positions of the mobile terminal is presented in Table I. The difference was found to be less than $1.0 \mathrm{~dB}$, indicating that both emulation techniques are equally capable of reproducing the throughput. This is in line with the findings from [9], where it is concluded through simulation that the spatial, polarimetric, and temporal dimensions of the target channel model can be created with both techniques.

\section{CONCLUSION}

In this letter, we compare two field emulation techniques, namely the PWS and PFS techniques, in the identical measurement setup of the multiprobe MIMO OTA chamber. The comparison is performed by measuring throughput, which is the actual FoM for the MIMO OTA testing. The measurements considered SCME UMi channel conditions at $806 \mathrm{MHz}$, which is the LTE lower band 20. A practical multielement mobile device is used as a test device. The throughput measurements for four different browsing positions of the mobile terminal showed that the differences of the DL power between the two emulation techniques were less than $1.0 \mathrm{~dB}$ at the median throughput with different browsing positions. Hence, both techniques are equally capable of emulating the target field inside the test zone and are technically suitable for MIMO OTA antenna testing using a chamber equipped with multiple probes.

\section{REFERENCES}

[1] "Verification of radiated multi-antenna reception performance of user equipment," 3rd Generation Partnership Project, Sophia Antipolis, France, Tech. Rep. 37.977 V12.1.0, Mar. 2014.

[2] P. Kyösti, J. Nuutinen, and T. Jämsä, "MIMO OTA test concept with experimental and simulated verification," in Proc. 4th Eur. Conf. Antennas Propag., Barcelona, Spain, Apr. 2010, pp. 1-5.

[3] W. Fan, X. Carreo, J. Nielsen, J. S. Ashta, G. F. Pedersen, and M. B. Knudsen, "Verification of emulated channels in multi-probe based MIMO OTA testing setup," in Proc. 7th Eur. Conf. Antennas Propag., Gothenburg, Sweden, Apr. 2013, pp. 97-101.

[4] W. Fan, "Over the air testing of MIMO capable terminals," Ph. D. dissertation, Aalborg Univ., Aalborg, Denmark, Oct. 2014.

[5] T. Laitinen, P. Kyösti, J.-P. Nuutinen, and P. Vainikainen, "On the number of OTA antenna elements for plane-wave synthesis in a MIMO-OTA test system involving a circular antenna array," in Proc. 4th Eur. Conf. Antennas Propag., Barcelona, Spain, Apr. 2010, pp. 1-5.

[6] J. Toivanen, T. Laitinen, V. Kolmonen, and P. Vainikainen, "Reproduction of arbitrary multipath environments in laboratory conditions," IEEE Trans. Instrum. Meas., vol. 60, no. 1, pp. 275-281, Jan. 2011.

[7] W. Fan, X. Carreo, J. Nielsen, K. Olesen, M. B. Knudsen, and G. F. Pedersen, "Measurement verification of plane wave synthesis technique based on multi-probe MIMO-OTA setup," in Proc. 76th IEEE Veh. Technol. Conf., Quebec City, Canada, 2012, pp. 1-5.

[8] M. Miah, A. Khatun, and K. Haneda, "Feasibility study of emulating extended spatial channel models in a multi-probe MIMO OTA antenna test setup," in Proc. Prog. Electromagn. Res. Symp., Prague, Czech Republic, Jul. 2015, pp. 1975-1980.

[9] P. Kyösti, T. Jämsä, and J. Nuutinen, "Channel modelling for multiprobe over-the-air MIMO testing," Int. J. Antennas Propag., vol. 2012, pp. 615954-1-615954-11, 2012.

[10] A. Khatun et al., "Experimental verification of a plane-wave field synthesis technique for MIMO OTA antenna testing," IEEE Trans. Antennas Propag., vol. 64, no. 7, pp. 3141-3150, Jul. 2016.

[11] "Test plan for $2 \times 2$ downlink MIMO and transmit diversity over-the-air performance," Cellular Telecommunications Industry Association, Washington, DC, USA, Version 1.1, Aug. 2016.

[12] "Spatial channel model for multiple input multiple output simulations," 3rd Generation Partnership Project, Sophia Antipolis, France, Tech. Rep. 25.996 V12.0.0, Sep. 2014.

[13] J. Hansen, Spherical Near-Field Antenna Measurement (IEE Electromagnetic waves series 26). London, U.K.: Peregrinus, 1998.

[14] M. Miah, A. Khatun, K. Haneda, L. Hentila, and A. Karilainen, "Effects of user's hand on the measurement setup in MIMO over-the-air testing," in Proc. 10th Eur. Conf. Antennas Propag., Davos, Switzerland, Apr. 2016, pp. 1-5. 